\title{
NOTAS
}

\section{Antecedentes dasométricos de Nothofagus alessandrii de la procedencia Coipué}

\author{
Forest mensuration of Nothofagus alessandrii of Coipué provenance
}

\author{
Rómulo Santelices $^{\mathrm{a}^{*}}$ y Marco Riquelme ${ }^{\mathrm{b}}$ \\ *Autor de correspondencia: aUniversidad Católica del Maule, Departamento de Ciencias Forestales, \\ Casilla 617, Talca, Chile, Fax 56-71-203524, rsanteli@ucm.cl \\ bUniversidad Católica del Maule, Instituto de Ciencias Básicas, Casilla 617, Talca, Chile.
}

\begin{abstract}
SUMMARY
Nothofagus alessandrii Espinosa is a species possessing unique characteristics, that grows only in the Coastal Cordillera of the Maule Region. Given its current distribution, N. alessandrii is currently listed as "in danger of extinction" by the Chilean government with around 350 ha remaining in a series of very fragmented stands. Despite the importance and conservation status of $N$. alessandrii, little information exists about this species, particularly in relation to its growth. In light of this, we conducted a study examining some of the forest mensuration of second growth stands in Coipue, near Gualleco in the Maule Region. The study was carried out by means of three temporal samples and on individual trees using stem analysis. Fifteen representative trees spanning the range of diameter, form and height were harvested. Forest mensuration variables were obtained from each tree. Step by step regression analysis was used to develop growth, volume and height functions. Results indicate that this stand is 57 years old with a density of 1,516 trees per hectare and $107 \mathrm{~m}^{2} \mathrm{ha}^{-1}$ of basal area. The growth, volume and height functions development are reliable. Diameter growth at the moment is $4.3 \mathrm{~mm}$ per year.
\end{abstract}

Key words: ruíl, functions, high, volume, growth.

\section{RESUMEN}

Nothofagus alessandrii es una especie con características muy particulares que crece sólo en algunos lugares de la Cordillera de la Costa en la Región del Maule. No obstante la importancia relativa que ha tenido la especie, actualmente se encuentra catalogada oficialmente como en peligro de extinción con un remanente de alrededor de 350 ha distribuidas en rodales muy fragmentados. A pesar de la importancia y del delicado estado de conservación existe poca información sobre esta especie, particularmente aquella que dice relación con el crecimiento. Por ello, se realizó un estudio tendiente a generar información dasométrica de rodales de segundo crecimiento del sector Coipué en las cercanías de Gualleco, comuna de Constitución, Región del Maule. Se efectuaron tres parcelas temporales de muestreo y un estudio a nivel del árbol individual por medio del análisis de tallo, para lo cual se cortaron 15 árboles representativos del rodal en cuanto a diámetro, forma y altura. Se obtuvieron tanto muestras de la especie como también información dasométrica de sus competidores. Por medio de análisis de regresión se desarrollaron funciones locales de volumen, altura y crecimiento. Los resultados indican que el rodal estudiado tiene a los 57 años de edad una densidad de 1.516 árboles ha $^{-1}$ y una ocupación de sitio de $107 \mathrm{~m}^{2} \mathrm{ha}^{-1}$. Las funciones locales de volumen y altura, más el modelo crecimiento diametral desarrollados son confiables. El crecimiento en diámetro arrojado por el modelo es, en promedio, 4,3 mm por año.

Palabras clave: ruíl, funciones, altura, volumen, crecimiento.

\section{INTRODUCCIÓN}

Nothofagus alessandrii Espinosa, conocido corrientemente como rúl, es una especie endémica de la zona central de Chile que se encuentra en peligro de extinción (Benoit 1989). Tiene un área de distribución natural muy restringida, limitada a la Cordillera de la Costa de la Región del Maule, en una faja que no supera los $100 \mathrm{~km}$ de extensión latitudinal, variando de los 100 a los 450 metros de altitud (San Martín et al. 1991). Es la especie más antigua de la familia de las Fagaceae del hemisferio sur (Serra et al. 1986), es decir, estos bosques son una de las formaciones más antiguas de Sudamérica. Forma parte de la asociación conocida como Bosque Maulino Costero y tiende a formar bosques puros en exposiciones de umbría. Es un árbol con una excelente forma que puede llegar a medir hasta $30 \mathrm{~m}$ de altura y $1 \mathrm{~m}$ de diámetro (Muñoz 1973, Rodríguez et al. 1983, Serra et al. 1986, 
Rodríguez y Quezada 2003). Su madera es de reconocida calidad, especialmente por su resistencia a la pudrición (Aravena y Molina 1976, Donoso 1983, Fierro y Pancel 1998), siendo posible encontrar en localidades cercanas a su distribución natural estacas de cerco que superan los 80 años conservadas en buen estado (Aravena y Molina 1976, Donoso 1983).

Las poblaciones remanentes se encuentran muy fragmentadas y están rodeadas por plantaciones de Pinus radiata D. Don, siendo ésta una especie agresiva con capacidad de invadir con éxito los bosques de ruíl (Bustamante y Grez 1995, Bustamante y Castor 1998). La superficie de los fragmentos es muy reducida y, en promedio, el $95 \%$ de ellos tiene una superficie inferior a las 12 ha (Bustamante y Grez 1995). Debido a la situación en que se encuentra esta especie, considerada incluso el árbol más amenazado de Chile, debería tener una alta prioridad de conservación (Hechenleitner et al. 2005), más aún cuando siguen presentes los procesos antropogénicos que la han llevado a su actual estado de degradación. En 12 años, la fragmentación ha aumentado en más de un 2,2\%, siendo el ser humano la principal causa de ello (Palma 2004).

Un elemento básico del manejo forestal sustentable, y obviamente de un programa de conservación, es contar con un diagnóstico que permita orientar la toma de decisiones. En este sentido, la mayor parte de los estudios sobre $N$. alessandrii se ha centrado en aspectos ecológicos que en detalle describen su crítica situación de conservación y sugieren la urgencia de efectuar acciones amplias para cambiar este estado (Bustamante y Castor 1998). Se ha constatado que estos bosques son de segundo crecimiento, originados de monte bajo en forma posterior a talas rasas (Donoso 1995), aunque se desconoce a plenitud cuántas veces las cepas se han vuelto a regenerar. En relación con el crecimiento de esta especie, aún existe un vacío de información (Olivares et al. 2005), y los aspectos más difundidos se circunscriben a los estudios realizados por Donoso (1978), quien señala que árboles regenerados por monte bajo con nueve años de edad alcanzaron alturas de $15 \mathrm{~m}$ y $25 \mathrm{~cm}$ de DAP. Por otra parte, Rodríguez et al. (1995) plantean que el crecimiento en altura puede llegar a cerca de $8 \mathrm{~m}$ a los 15 años de edad. Otros estudios sobre el particular señalan que después de un raleo es posible obtener un incremento anual de más de $11 \mathrm{~m}^{3} \mathrm{ha}^{-1}$ en un período de ocho años (Deus y Bravo 1984, San Martín et al. 1991). Existe también información sobre el crecimiento de plantas cultivadas en vivero, con resultados que, aunque variables, muestran crecimientos superiores a los $35 \mathrm{~cm}$ en altura y a los $4 \mathrm{~mm}$ de diámetro de cuello para plantas del tipo 1/0 (San Martín et al. 2006).

En general, la información que actualmente se dispone es insuficiente para tomar decisiones que permitan cambiar, al menos en el corto plazo, la situación de conservación en que se encuentra esta especie. En particular, los antecedentes existentes sobre crecimiento no cubren todas las áreas en que se distribuye naturalmente la especie y, en consecuencia, cualquier información que ayude a aumentar el estado actual del conocimiento en este aspecto es un aporte valioso. Contar con antecedentes dasométricos permitiría proyectar modelos silvícolas tendientes a revalorizar e incrementar el recurso, de manera de asegurar su conservación.

En consideración con lo anterior, el objetivo de este trabajo es generar funciones locales de volumen y altura, y un modelo de crecimiento diametral para un rodal representativo de $N$. alessandrii de la procedencia Coipué.

\section{MÉTODOS}

Ubicación y características del rodal estudiado. Se seleccionó un rodal característico de la procedencia Coipué, ubicado en las cercanías del pueblo de Gualleco, en la Región del Maule ( $35^{\circ} 16^{\prime}$ S y $72^{\circ} 08^{\prime} \mathrm{O}$, a $412 \mathrm{~m}$ de elevación) (figura 1). Debido a las restricciones que tiene la especie desde el punto de vista de su conservación, no fue posible contar con la autorización para intervenir otros rodales de ésta.

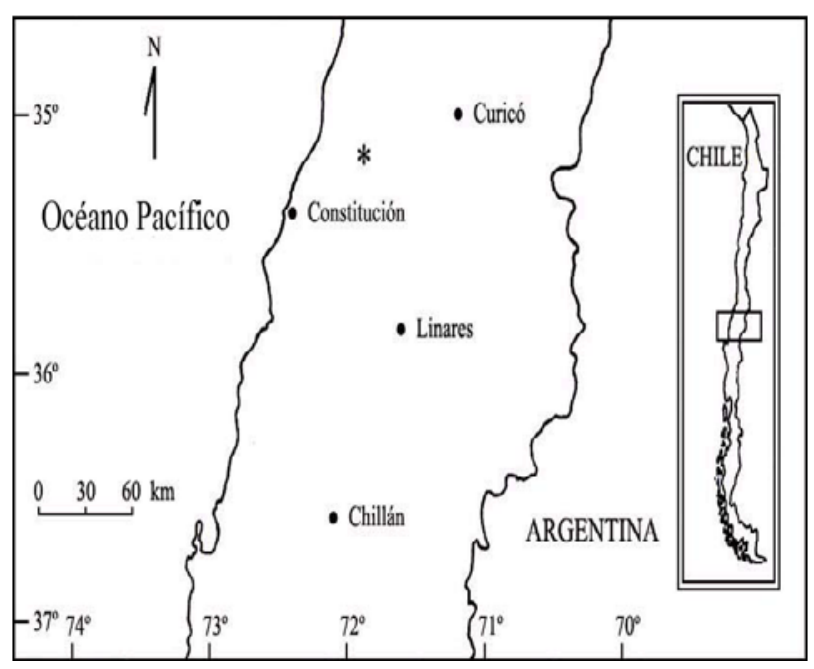

Figura 1. Mapa de la zona central de Chile que describe el área de estudio (*) en la Cordillera de la Costa.

Map of south-central Chile depicting the study area $(*)$ in the Coastal Cordillera.

El rodal representa una típica formación del subtipo forestal "Bosquetes de ruíl" (Donoso 1995) y está rodeado por plantaciones de $P$. radiata. Tiene una extensión de 2,1 ha en exposición de umbría y una topografía ondulada con pendientes superiores al $50 \%$. Es un bosque puro regenerado por monte bajo y las principales especies leñosas acompañantes son Persea lingue (Ruiz et Pav.) Nees, Cryptocaria alba (Mol.) Looser, N. glauca (Phil.) Krasser, Quillaja saponaria Mol. y Peumus boldus Mol. 
Muestreo para el análisis de árboles individuales. Con el objeto de conocer el desarrollo de las variables de estado y construir los modelos de crecimiento, se realizaron tres parcelas temporales de $200 \mathrm{~m}^{2}$ cada una. En cada unidad de muestreo se seleccionaron varios individuos, que se denominaron "árboles sujeto", de distintas clases de edad, altura y espaciamiento. Una vez identificados los árboles sujeto, se seleccionaron y registraron los árboles que ejercían la competencia más directa sobre ellos. Por restricciones en la cantidad de árboles disponibles de diferentes diámetros, sólo se seleccionaron 15 árboles sujeto, intentando abarcar la mayor distribución diamétrica.

La información obtenida en terreno para los árboles sujeto y sus competidores fue la siguiente: diámetro a la altura del pecho (DAP a $1,3 \mathrm{~m}$ ), altura total, altura del inicio de la copa, radios de la copa (de acuerdo a los puntos cardinales) y distancia entre los árboles sujeto y sus competidores.

Posteriormente, los árboles sujeto se voltearon y trozaron cada dos metros y medio. Se obtuvieron rodelas de la base del árbol, de la sección mayor de cada troza, hasta un índice de utilización de ocho centímetros y una a la altura del DAP. De cada troza se obtuvo, además, el diámetro en cada extremo a fin de generar la información necesaria para elaborar las funciones de volumen. Luego las rodelas fueron identificadas y embaladas por cada árbol $\mathrm{y}$ transportadas al laboratorio.

Procesamiento de la información dasométrica. Con el objeto de visualizar en forma óptima los anillos de crecimiento fue necesario pulir las rodelas. Para conseguir esto, se redujo su contenido de humedad por debajo del punto de saturación de las fibras. Con este fin, las rodelas se mantuvieron en un secador convencional entre 50 y $60^{\circ} \mathrm{C}$ durante un periodo de 15 días. El contenido de humedad, medido con xilohigrómetro, alcanzó en promedio un $20 \%$.

Una vez lijadas las rodelas, se realizaron mediciones en cuatro radios orientados en las direcciones norte, sur, este y oeste (Kramer y Akça 1982, Cubillos 1987, Grosse y Kannegiesser 1988, Santelices 1989).

Se efectuó un análisis de tallo utilizando la gráfica de la curva de ahusamiento de los árboles selectos. El procesamiento de la información se realizó mediante el ajuste de una función múltiple, que permite estimar los parámetros del árbol en función de la edad, diámetro a la altura del pecho, área basal, altura total, volumen cúbico y sus respectivos crecimientos.

En la construcción de las funciones de volumen cúbico total se utilizó el análisis de regresión entre las variables independientes DAP y altura total, y la variable dependiente volumen cúbico total sin corteza. La muestra utilizada para el cálculo del volumen corresponde a los mismos árboles que se ocuparon para el análisis del tallo. Además, con la información registrada en las tres parcelas de muestreo se ajustó un modelo local de altura, utilizando un total de 49 árboles entre los denominados "sujetos" y "competidores".

De acuerdo con los antecedentes bibliográficos acerca de la estimación del volumen y de la altura, existen varias funciones comúnmente utilizadas (Prodan 1965, Loetsch $e t$ al. 1973, Zöhrer 1980). El criterio adoptado para la selección de modelos a probar se fundamenta en su bondad de ajuste y su mejor aplicación práctica (Zöhrer 1980).

Para el cálculo del volumen por troza, se empleó la fórmula de Smalian (Husch et al. 1982). Se utilizaron trozas con un diámetro mínimo de $8 \mathrm{~cm}$ sin corteza. Posteriormente se ajustó la variable DAP con el volumen de cada troza, a través de los modelos usualmente empleados.

La construcción de los modelos de crecimiento diametral se realizó sobre la base de las muestras sometidas a análisis de tallo. Se estableció como variable independiente el incremento diametral anual periódico (IDAPA en $\mathrm{mm}$ ).

Las variables independientes que se consideran provienen del árbol sujeto y de sus competidores, y se describen a continuación:

DAP : diámetro a la altura del pecho (a 1,3 $\mathrm{m}$ de altura, en $\mathrm{cm}$ ).

HT : altura total (en m).

HIC : altura inicio de copa (en m).

VT : volumen total (en $\left.\mathrm{m}^{3} \mathrm{ssc}\right)$.

DC : diámetro de copa (en m).

DISME : distancia media a los árboles competidores (en $\mathrm{m}$ ).

DAPCO: DAP medio de los competidores (a 1,3 m de altura, en $\mathrm{cm}$ ).

HTCO : altura total media de los competidores (en m). HICCO : altura de inicio de copa media de los competidores (en m).

DCCO : diámetro de copa media de los competidores (en $\mathrm{m}$ ).

E : edad (años)

La bondad de ajuste de los modelos de regresión se evaluó con el coeficiente de determinación $\left(\mathrm{R}^{2}\right)$ y el cuadrado medio del error (CME). Se incorpora, además, el valor $P$ ( $p$-value) asociado al análisis de varianza para chequear si el ajuste es significativo.

Para seleccionar un modelo de regresión adecuado se efectuó un análisis de regresión paso a paso y también un análisis denominado método de todas las regresiones posibles (se ajustaron 2.048 funciones) que considera los criterios de $\mathrm{R}^{2}$, CME y $\mathrm{C}_{\mathrm{p}}$ de Mallows (Walpole et al. 1999). Mediante el software estadístico MINITAB se obtuvieron los subconjuntos óptimos de variables independientes para ser consideradas en el modelo de regresión.

Situación de estado del rodal estudiado. El rodal presentó 1.516 árboles ha ${ }^{-1}$, DAP promedio de $28 \mathrm{~cm}$, diámetro medio cuadrático de $30 \mathrm{~cm}$, área basal de $107 \mathrm{~m}^{2} \mathrm{ha}^{-1}$ y altura total promedio de $19 \mathrm{~m}$. Una tabla de rodal se 
entrega en el cuadro 1. Estos antecedentes corresponden al promedio de las tres parcelas temporales de muestreo y son representativos del rodal y de la zona.

Cuadro 1. Tabla de rodal representativa del lugar de estudio. Representative stand table of the study area.

\begin{tabular}{cc}
\hline DAP $(\mathrm{cm})$ & Densidad (árboles ha $\left.{ }^{-1}\right)$ \\
\hline 16 & 183 \\
20 & 250 \\
24 & 133 \\
28 & 217 \\
32 & 217 \\
36 & 150 \\
40 & 100 \\
44 & 150 \\
48 & 83 \\
52 & 33 \\
\hline Total & 1.516 \\
\hline
\end{tabular}

\section{RESULTADOS}

Funciones de volumen local. Los mejores resultados obtenidos fueron los siguientes:

$$
V=-0,007899043+0,000036 * D^{2} * H
$$

Donde $V=$ volumen total del árbol en $\mathrm{m}^{3} \mathrm{ssc}, D=\mathrm{DAP}$ en $\mathrm{cm}, H=$ altura en $\mathrm{m}\left(\mathrm{R}^{2}=0,94 ; \mathrm{CME}=0,005 ; \mathrm{n}=15\right.$; $P \approx 0,000)$.

$$
V=-0,054967192+0,0008176 * D^{2}
$$

Donde $V=$ volumen total del árbol en $\mathrm{m}^{3} \mathrm{ssc}, D=\mathrm{DAP}$ en $\mathrm{cm}\left(\mathrm{R}^{2}=0,92 ; \mathrm{CME}=0,006 ; \mathrm{n}=15 ; P \approx 0,000\right)$.

Las funciones de volumen obtenidas son bastante confiables de acuerdo a los parámetros utilizados para medir la bondad del ajuste de las regresiones. Se debe tener en cuenta que sólo son representativas para el área específica donde se realizó el muestreo y, en consecuencia, sería interesante generar funciones para otras zonas y compararlas. La función [2] tiene la ventaja de ser más fácil de usar al no considerar la altura como variable.

Funciones locales de altura. Los mejores resultados obtenidos se presentan a continuación:

$$
\begin{gathered}
H=4,4486496+1,3974164 * D \\
-0,0443592 * D^{2}+0,00052455 * D^{3}
\end{gathered}
$$

Donde $H=$ altura en del árbol en m, $D=$ DAP en $\mathrm{cm}$ $\left(\mathrm{R}^{2}=0,74, \mathrm{CME}=1,12, \mathrm{n}=49, P \approx 0,000\right)$.

$$
H=11,6199829+0,4214651 * D-0,0037059 * D^{2}
$$

Donde $H=$ altura en del árbol en $\mathrm{m}, D=\mathrm{DAP}$ en $\mathrm{cm}$ $\left(\mathrm{R}^{2}=0,72 ; \mathrm{CME}=1,20 ; \mathrm{n}=49 ; P \approx 0,000\right)$.

Los dos modelos propuestos tienen una explicación mayor a un $72 \%$ de la variación en altura. Esto se debe, probablemente, al hecho de tratarse de un bosque regenerado por monte bajo.

Modelos de crecimiento diametral. Los modelos de regresión ajustados más apropiados para estimar el incremento diametral periódico anual son los que se presentan a continuación:

$$
\begin{gathered}
I D A P A=\quad 0,11515+0,78534 * H T \\
-0,37074 * H I C-0,33751 * D A P \\
\begin{array}{r}
\left(\mathrm{R}^{2}=0,73 ; \mathrm{CME}=0,57 ; \mathrm{n}=15 ; P=0,002\right) . \\
I D A P A=-6,2967+0,76678 * H T-0,37441 * H I C \\
\quad-0,30377 * D A P+2,62184 * D C C O
\end{array} \\
\left(\mathrm{R}^{2}=0,78 ; \mathrm{CME}=0,51 ; \mathrm{n}=15 ; P=0,0024\right) .
\end{gathered}
$$

Donde: $I D A P A=$ incremento diametral periódico anual en $\mathrm{mm}, H T=$ altura total en $\mathrm{m}, H I C=$ altura del inicio de la copa en $\mathrm{m}, D A P=$ diámetro a $1,3 \mathrm{~m}$ en $\mathrm{cm}$, $D C C O=$ diámetro medio de copa de los competidores en $\mathrm{m}$.

A través del método de todas las regresiones posibles se confirmó que el modelo dado en [5] es adecuado, siendo altamente significativo $(P<0,01)$. El esquema entregado por el software estadístico MINITAB (cuadro 2) muestra el análisis de los subconjuntos óptimos de variables independientes para ser consideradas en el modelo de regresión. Se puede apreciar en la quinta fila que el modelo dado en [5] es razonable, pues considera pocas variables independientes y a la vez valores apropiados de $\mathrm{R}^{2}$, CME y $\mathrm{C}_{\mathrm{p}}$ de Mallows (criterio de parsimonia).

Para todas las funciones desarrolladas, el rango de las variables utilizadas correspondiente a los árboles muestra fue el siguiente: DAP de 16 a $34 \mathrm{~cm}$, la altura total de 17,5 a 22,9 m, el volumen del árbol de 0,139 a 0,873 $\mathrm{m}^{3} \mathrm{ssc}$, la altura del inicio de la copa de los 6 a los 14 m, la edad de los árboles de 33 a 82 años, el diámetro medio de copa de 1 a $8,4 \mathrm{~m}$, la distancia media a los árboles competidores de 1,9 a $6,5 \mathrm{~m}$, y el incremento anual periódico de los últimos 10 años de 2,3 a 6,6 mm. Para los árboles catalogados como competidores, el rango fue el siguiente: el DAP de 16,1 a $24,6 \mathrm{~cm}$, la altura de 12,8 a $19,6 \mathrm{~m}$, la altura del inicio de la copa de 5,4 a 9,7 m y el diámetro medio de copa de 2 a 2,5 m. 
Cuadro 2. Análisis de los subconjuntos óptimos de las variables independientes.

Analysis of optimal subsets of independent variables.

\begin{tabular}{|c|c|c|c|c|c|c|c|c|c|c|c|c|c|c|}
\hline Variables & $\mathrm{R}^{2}$ & CME & $\mathrm{C}_{\mathrm{p}}$ & $\frac{a}{\Delta}$ & 吉 & $\begin{array}{l}\vec{Z} \\
\text { II }\end{array}$ & 5 & $\underline{\Xi}$ & Uִ & 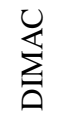 & 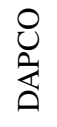 & $\begin{array}{l}0 \\
\text { 王 }\end{array}$ & $\begin{array}{l}0 \\
\text { 己 }\end{array}$ & $\begin{array}{l}0 \\
\text { U }\end{array}$ \\
\hline 1 & 0,27 & 1,31 & 31,7 & - & - & - & - & - & - & - & $\mathrm{x}$ & - & - & - \\
\hline 1 & 0,23 & 1,37 & 33,8 & - & - & - & - & - & - & - & - & - & - & $\mathrm{x}$ \\
\hline 2 & 0,55 & 0,88 & 17,5 & $\mathrm{x}$ & $\mathrm{x}$ & - & - & - & - & - & - & - & - & - \\
\hline 2 & 0,50 & 0,98 & 20,4 & - & $\mathrm{x}$ & - & $\mathrm{x}$ & - & - & - & - & - & - & - \\
\hline 3 & 0,73 & 0,57 & 8,7 & $\mathrm{x}$ & $\mathrm{x}$ & - & - & $\mathrm{x}$ & - & - & - & - & - & - \\
\hline 3 & 0,62 & 0,80 & 15,0 & - & $\mathrm{x}$ & - & $\mathrm{x}$ & - & - & - & - & - & - & $\mathrm{x}$ \\
\hline 4 & 0,78 & 0,51 & 7,8 & $\mathrm{x}$ & $\mathrm{x}$ & - & - & $\mathrm{x}$ & - & - & - & - & - & $\mathrm{x}$ \\
\hline 4 & 0,77 & 0,53 & 8,4 & $\mathrm{x}$ & $\mathrm{x}$ & - & - & $\mathrm{x}$ & - & $\mathrm{x}$ & - & - & - & - \\
\hline 5 & 0,82 & 0,47 & 7,6 & $\mathrm{x}$ & $\mathrm{x}$ & - & - & $\mathrm{x}$ & - & $\mathrm{x}$ & - & - & - & $\mathrm{x}$ \\
\hline 5 & 0,81 & 0,50 & 8,3 & $\mathrm{x}$ & $\mathrm{x}$ & - & - & $\mathrm{x}$ & - & - & $\mathrm{x}$ & - & - & $\mathrm{x}$ \\
\hline 6 & 0,83 & 0,50 & 9,0 & $\mathrm{x}$ & $\mathrm{x}$ & - & - & $\mathrm{x}$ & - & - & $\mathrm{x}$ & $\mathrm{x}$ & - & $\mathrm{x}$ \\
\hline 6 & 0,82 & 0,51 & 9,3 & $\mathrm{x}$ & $\mathrm{x}$ & $\mathrm{x}$ & - & $\mathrm{x}$ & - & - & $\mathrm{x}$ & $\mathrm{x}$ & - & - \\
\hline 7 & 0,86 & 0,48 & 9,4 & $\mathrm{x}$ & $\mathrm{x}$ & - & - & $\mathrm{x}$ & - & - & $\mathrm{x}$ & $\mathrm{x}$ & $\mathrm{x}$ & $\mathrm{x}$ \\
\hline 7 & 0,84 & 0,54 & 10,5 & $\mathrm{x}$ & $\mathrm{x}$ & $\mathrm{x}$ & - & $\mathrm{x}$ & - & - & $\mathrm{x}$ & $\mathrm{x}$ & - & $\mathrm{x}$ \\
\hline 8 & 0,88 & 0,46 & 9,9 & $\mathrm{x}$ & $\mathrm{x}$ & - & - & $\mathrm{x}$ & - & $\mathrm{x}$ & $\mathrm{x}$ & $\mathrm{x}$ & $\mathrm{x}$ & $\mathrm{x}$ \\
\hline 8 & 0,86 & 0,53 & 10,9 & $\mathrm{x}$ & $\mathrm{x}$ & - & $\mathrm{x}$ & $\mathrm{x}$ & - & - & $\mathrm{x}$ & $\mathrm{x}$ & $\mathrm{x}$ & $\mathrm{x}$ \\
\hline 9 & 0,90 & 0,45 & 10,7 & $\mathrm{x}$ & $\mathrm{x}$ & $\mathrm{x}$ & - & $\mathrm{x}$ & - & $\mathrm{x}$ & $\mathrm{x}$ & $\mathrm{x}$ & $\mathrm{x}$ & $\mathrm{x}$ \\
\hline 9 & 0,89 & 0,50 & 11,3 & $\mathrm{x}$ & $\mathrm{x}$ & - & $\mathrm{x}$ & $\mathrm{x}$ & - & $\mathrm{x}$ & $\mathrm{x}$ & $\mathrm{x}$ & $\mathrm{x}$ & $\mathrm{x}$ \\
\hline 10 & 0,94 & 0,33 & 10,3 & $\mathrm{x}$ & $\mathrm{x}$ & $x$ & - & $\mathrm{x}$ & $\mathrm{x}$ & $\mathrm{x}$ & $\mathrm{x}$ & $\mathrm{x}$ & $\mathrm{x}$ & $\mathrm{x}$ \\
\hline 10 & 0,92 & 0,46 & 11,6 & $\mathrm{x}$ & $\mathrm{x}$ & $x$ & $\mathrm{x}$ & $\mathrm{x}$ & - & $\mathrm{x}$ & $\mathrm{x}$ & $\mathrm{x}$ & $\mathrm{x}$ & $\mathrm{x}$ \\
\hline 11 & 0,95 & 0,40 & 12,0 & $\mathrm{x}$ & $\mathrm{x}$ & $\mathrm{x}$ & $\mathrm{x}$ & $\mathrm{x}$ & $\mathrm{x}$ & $\mathrm{x}$ & $\mathrm{x}$ & $\mathrm{x}$ & $\mathrm{x}$ & $\mathrm{x}$ \\
\hline
\end{tabular}

\section{DISCUSIÓN}

Funciones locales de volumen y de altura. De acuerdo a los indicadores utilizados para estimar la bondad de ajuste, las funciones de volumen obtenidas para el área de Coipué son bastante confiables y son las únicas generadas hasta ahora para la especie. Al compararlas con otras desarrolladas para especies del mismo género (Drake et al. 2003), los volúmenes entregados por árbol individual son similares. Por ejemplo, la diferencia con Nothofagus alpina (Poepp. et Endl.) Oerst para la zona costera de la Región del Bío Bío, considerando como variable independiente el DAP al cuadrado (Cubillos 1987), para un diámetro de $25 \mathrm{~cm}$, es de alrededor de un $10 \%$ mayor al volumen entregado por la función desarrollada para $N$. alessandrii. Al comparar con $N$. alpina de la zona precordillerana de la Región del Maule, considerando también un diámetro de $25 \mathrm{~cm}$ y una altura de $18 \mathrm{~m}$, y como variables independientes el DAP al cuadrado multiplicado por la altura del árbol (Drake et al. 2003), la diferencia aumenta a cerca de un $15 \%$. Probablemente el mayor volumen entregado por árbol individual para $N$. alessandrii pueda explicarse por una mejor forma de los árboles muestreados para esta especie.

Los modelos seleccionados para las funciones de altura explican cerca de la mitad de la variación en altura, razón por la cual es recomendable considerarlos sólo como referencia. Sin embargo, sería interesante desarrollar otros modelos y compararlos con los propuestos.

Modelos de crecimiento diametral. La muestra para construir el modelo de incremento diametral se obtuvo del dosel superior del rodal, estrato dominado por $N$. alessandrii, lo que coincide con lo señalado por Olivares et al. (2005). De acuerdo a los parámetros utilizados para medir la bondad del ajuste, las funciones desarrolladas son confiables y representan fielmente la situación del rodal. Las variables que explican los dos modelos propuestos son el DAP, la altura total, la altura del inicio de la copa 
de los árboles muestra y el diámetro medio de la copa de los competidores. Los dos modelos no consideran la edad del árbol, lo que sí hacen la mayoría de las funciones de crecimiento diametral propuestas para especies del mismo género (Drake et al. 2003). En el proceso de análisis de los datos no se observó una correlación entre la edad, el DAP y el crecimiento de los árboles.

$\mathrm{Al}$ aumentar la altura del árbol, también lo hace el incremento diametral, lo que podría indicar que desde un punto de vista fotosintético las copas podrían ser más eficientes al estar más liberadas. Se ha planteado que $N$. alessandrii podría ser una especie poco tolerante a la competencia (San Martín et al. 1984). En este mismo sentido, a mayor altura del inicio de la copa, lo que indicaría copas más pequeñas, disminuye el crecimiento diametral. A medida que aumenta el diámetro del árbol es menor su crecimiento, probablemente por tratarse de árboles maduros, regenerados muchas veces por monte bajo.

El mejor modelo seleccionado [5] no considera las variables relacionadas con los árboles competidores y el otro modelo [6] sólo lo hace con el diámetro de copa. La escasa influencia de la competencia en los modelos desarrollados probablemente esté explicada porque el rodal es prácticamente puro y la vegetación acompañante se encuentra en un dosel inferior al que está dominado por $N$. alessandrii, situación que es característica de los bosques de esta especie (Olivares et al. 2005). En efecto, las especies acompañantes son $P$. lingue, $C$. alba, N. glauca, Q. saponaria y P. boldus; todas ellas, a excepción de $N$. glauca, habitan en un piso inferior al dominado por $N$. alessandrii. En promedio, la altura de los árboles competidores fue de 17,2 $\pm 0,1 \mathrm{~m}$, en cambio la de los árboles muestra fue de 20,2 $\mathrm{m} \pm 0,1 \mathrm{~m}$, lo que indicaría que los árboles muestra producto de su posición fitosociológica no tendrían competencia por luz directa.

El crecimiento medio anual en diámetro que entrega el modelo es de $4,3 \mathrm{~mm}$, lo que puede considerarse bajo si se compara con algunos valores observados macroscópicamente en las muestras, que llegaron hasta los 6,6 $\mathrm{mm}$. Esto podría ser un indicador de que el rodal, a pesar de tener la capacidad de regenerarse, no tendría el vigor de un material más joven. Por otra parte, es claro que un rodal prácticamente puro, de 57 años de edad y que tiene una densidad de 1.516 árboles ha $^{-1}$, se encontraría desarrollando una alta competencia intraespecífica y bajo esas condiciones es poco probable que pueda tener una tasa de crecimiento mayor.

Se ha documentado que árboles regenerados por monte bajo a los nueve años de edad alcanzaron $25 \mathrm{~cm}$ de diámetro (Donoso 1978), lo que es un crecimiento mucho mayor al observado en el rodal estudiado y probablemente se deba a condiciones de sitio diferentes y a cepas con mayor vigor. En rodales de entre 60 y 70 años de edad de la zona de Empedrado, área cercana al lugar de estudio, San Martín et al. (1991) evaluaron el efecto de un raleo después de ocho años. Los resultados muestran que con todos los tratamientos el rodal mostró una reacción positiva a la intervención, alcanzando en el periodo de ocho años un incremento anual de $0,75 \mathrm{~m}^{2} \mathrm{ha}^{-1}$ (extrayendo un $21 \%$ del área basal y dejando un remanente de $16 \mathrm{~m}^{2} \mathrm{ha}^{-1}$ ). Como se puede apreciar, el crecimiento medio entregado por el modelo no es comparable con los señalados por Donoso (1995) y San Martín et al. (1991), básicamente por tratarse de condiciones de sitio y de rodal diferentes.

Es probable que los resultados de crecimiento arrojados por el modelo propuesto no reflejen el verdadero potencial que tiene esta especie y, en consecuencia, es recomendable estudiar su desarrollo con material vegetal más joven o en condiciones en que la competencia no sea tan marcada. En este marco, sería interesante estudiar el crecimiento de rodales jóvenes, para lo cual debería considerarse la reposición de las actuales cepas, ya sea mediante regeneración natural a partir del mismo rodal o directamente por medio de la plantación.

\section{CONCLUSIONES}

Las funciones generadas son de carácter local y, en consecuencia, sólo describen la situación particular para la procedencia Coipué. De acuerdo a los parámetros estadísticos empleados en su elaboración se puede decir que son confiables y aceptables.

Para un rodal con una densidad de 1.516 árboles por hectárea, a los 57 años de edad se tiene un incremento diametral anual medio de $4,3 \mathrm{~mm}$.

\section{AGRADECIMIENTOS}

Se agradece la ayuda prestada en los trabajos de campo y procesamiento de la información a los Sres. Paulo Bascuñán y Mauricio Escobar, y a la Corporación Nacional Forestal (CONAF) por el apoyo brindado a esta investigación.

\section{REFERENCIAS}

Aravena P, V Molina. 1976. Los Robles-Nothofagus de la Séptima Región de Chile. UC Maule (Chile) 3(1): 13-18.

Benoit I. 1989. Libro rojo de la flora terrestre de Chile (Primera parte). Santiago, Chile. Corporación Nacional Forestal. $157 \mathrm{p}$.

Bustamante R, A Grez. 1995. Consecuencias ecológicas de la fragmentación de los bosques nativos. Ambiente y Desarrollo (Chile) 11(2): 58-63.

Bustamante R, C Castor. 1998. The decline of an endangered ecosystem: the ruil (Nothofagus alessandrii) forest in Central Chile. Biodiversity and Conservation 7: 1607-1626.

Cubillos V. 1987. Modelos de crecimiento diametral para algunos renovales de raulí. Ciencia e Investigación Forestal (Chile) 1(1): 68-76. 
Deus R, R Bravo. 1984. Ensayo de Raleo, Regeneración y Semillación en Renovales de Ruíl. Primera Parte: Instalación, Inventarios y Resultados Preliminares. Talca, Chile. Corporación Nacional Forestal 135 p.

Donoso C. 1978. Dendrología: Árboles y arbustos chilenos. Boletín Técnico $\mathrm{N}^{\circ}$ 2. Santiago. Chile. Universidad de Chile. 142 p.

Donoso C. 1983. Árboles nativos de Chile: guía de reconocimiento. Valdivia, Chile. Marisa Cuneo Ediciones. 116 p.

Donoso C. 1995. Bosques templados de Chile y Argentina: variación, estructura y dinámica. Santiago, Chile. Editorial Universitaria. 483 p.

Drake F, P Emannuelli, E Acuña. 2003. Compendio de funciones dendrométricas del bosque nativo. Santiago, Chile. CONAF y GTZ. 197 p.

Fierro S, L Pancel. 1998. Experiencia Silvicultural del Bosque Nativo de Chile, recopilación de 57 especies arbóreas y evaluación de prácticas silviculturales. Proyecto Manejo Sustentable del Bosque Nativo. Santiago, Chile. CONAF y GTZ. 420 p.

Grosse H, U Kannegiesser. 1988. Investigación para el manejo de las plantaciones de pino oregón y Sequoia sempervirens (Informe Final). Concepción, Chile. Instituto Forestal. $179 \mathrm{p}$.

Hechenleitner P, M Gardner, P Thomas, C Echeverría, B Escobar, P Brownless, C. Martínez. 2005. Plantas Amenazadas del Centro-Sur de Chile. Distribución, Conservación y Propagación. Valdivia, Chile. Universidad Austral de Chile y Real Jardín Botánico de Edimburgo. 188 p.

Husch B, CH Miller, T Beers. 1982. Forest mensuration. Canada. John Wiley. 401 p.

Kramer H, A Akça. 1982. Leitfäden für Dendrometrie und Bestandesinventur. Frankfurt a. M., Germany. Sauerländer's Verlag. 251 p.

Loetsch F, F Zöhrer, K Haller. 1973. Forest inventory. Vol. II. Munich, Germany. BLV. 467 p.

Muñoz C. 1973. Chile: plantas en extinción. Santiago, Chile. Universitaria. $248 \mathrm{p}$.

Olivares P, J San Martín, R Santelices. 2005. Ruíl (Nothofagus alessandrii): Estado del Conocimiento y Desafíos para su Conservación. Talca, Chile. Comisión Nacional del Medio Ambiente (CONAMA). 48 p.
Palma K. 2004. Evaluación de la degradación de los fragmentos vegetacionales de Nothofagus alessandrii, "ruíl", en el período 1991-2003, en la VII Región del Maule. Tesis Ingeniero Forestal. Talca, Chile. Facultad de Ciencias Forestales, Universidad de Talca. 89 p.

Prodan M. 1965. Holzmesslehre. Frankfurt. a. M. Germany. Sauerländer's Verlag. 644 p.

Rodríguez R, O Matthei, M Quezada. 1983. Flora arbórea de Chile. Concepción, Chile. Universidad de Concepción. 408 p.

Rodríguez G, R Rodríguez, H Barrales. 1995. Plantas ornamentales chilenas, 100 árboles, arbustos y trepadoras. Concepción, Chile. Lamas. 230 p.

Rodríguez R, M Quezada. 2003. Fagaceae. In Marticorena C, R Rodríguez eds. Flora de Chile Vol. 2 Fasc. 2 (Berberidaceae - Betulaceae). Concepción, Chile. Universidad de Concepción. p. 64-76.

Santelices R. 1989. Funciones de volumen, factores de forma y modelos de crecimiento diametral para rodales de lingue y mañío. Ciencia e Investigación Forestal (Chile) 3(2): 1-19.

San Martín J, H Figueroa, C Ramírez. 1984. Fitosociología de los bosques de ruíl (Nothofagus alessandrii Espinosa) en Chile Central. Revista Chilena de Historia Natural 57: 171-200.

San Martín J, V Mourgues, A Villa, C Carreño. 1991. Catastro actualizado de la distribución y estado de conservación de los bosques de ruíl en la VII Región. Informe Final Proyecto Investigación y Desarrollo Forestal CHI/89/003. Talca, Chile. Corporación Nacional Forestal. 35 p.

San Martín J, R Santelices, R Henríquez. 2006. Nothofagus alessandrii Espinosa, Ruíl. Familia: Fagaceae. In Donoso $\mathrm{C}$ ed. Las especies arbóreas de los bosques templados de Chile y Argentina. Autoecología. Valdivia, Chile. Marisa Cuneo Ediciones. p. 390-400.

Serra MT, R Gajardo, A Cabello. 1986. Programa de protección y recuperación de la flora nativa de Chile, Ficha Técnica de Especies Amenazadas: Nothofagus alessandrii Espinosa. Santiago, Chile. Corporación Nacional Forestal. 32 p.

Walpole R, R Myers, S Myers. 1999. Probabilidad y estadística para ingenieros. México DF, México. $6^{\mathrm{a}}$ ed. Prentice Hall. $450 \mathrm{p}$.

Zöhrer F. 1980. Forstinventur. Berlin, Hamburg, Germany. Paul Parey. 207 p. 